\title{
High Resolution Probe for filament transport and current density study at the edge region of W7-X
}

\author{
M. Spolaore, ${ }^{a 1}$ P. Agostinetti, ${ }^{a}$ C. Killer, ${ }^{b}$ M. Moresco, ${ }^{a}$ M. Brombin, ${ }^{a}$ R. Cavazzana, ${ }^{a}$ R. \\ Ghirardelli, $^{a}$ G. Grenfell, ${ }^{a}$ O. Grulke, ${ }^{b}$ S. A. Lazerson, ${ }^{c}$ E. Martines, ${ }^{a}$ O. Neubauer, ${ }^{d}$ D. \\ Nicolai,$^{d}$ G. Satheeswaran, ${ }^{d}$ B. Schweer, ${ }^{d}$ N. Vianello,${ }^{a}$ M. Visentin , ${ }^{a}$ and W7-X team \\ ${ }^{a}$ Consorzio RFX (CNR, ENEA, INFN, Università di Padova, Acciaierie Venete SpA), Padova, Italy \\ ${ }^{b}$ Max-Planck-Institut für Plasmaphysik, Greifswald, Germany \\ ${ }^{c}$ Princeton Plasma Physics Laboratory, Princeton NJ, 08543, USA \\ ${ }^{d}$ Forschungszentrum Jülich GmbH, Institut für Energie- und Klimaforschung Plasmaphysik, Partner of \\ the Trilateral Euregio Cluster (TEC), 52425 Jülich, Germany
}

E-mail: monica.spolaore@igi.cnr.it

AbSTRACT: For the study of electrostatic and magnetic properties of filaments characterizing the edge region of the stellarator experiment $\mathrm{W} 7-\mathrm{X}$, a specifically designed insertable probe head was constructed within the framework of EUROfusion WP.S1 work package in collaboration between Consorzio RFX, IPP Greifswald and Forschungszentrum Jülich. The probe head, named High Resolution Probe (HRP), was conceived to be installed on the mid-plane multi-purpose fast reciprocating manipulator on W7-X. Electromagnetic filamentary turbulent structures are found to characterize the edge region of different magnetic configurations including Reversed Field Pinch, stellarator and tokamak, where strong currents are associated also to ELM filamentary structures. The study of those phenomena in W7-X stellarator is of particular interest as the electromagnetic features of filaments are expected to become more relevant with the increase of the local plasma beta. In particular the aim is to provide information about the presence and the features of parallel current density associated to filamentary turbulent or ELM-like structures. Furthermore, the possibility to measure the time evolution of the flow radial profiles using the Mach probe array was considered as a further interesting part of the study, given the strong interplay expected between the turbulent fluctuation and the average flows. Further important information provided is the radial propagation of turbulent flux. The contribution will present the design development, the $R \& D$ studies and the applied solutions for the sensors embedded in the probe head. In particular, the presence of 140 $\mathrm{GHz}$ ECRH plasma environment represents one of the main challenges for reliable magnetic fluctuation measurements. First measurements were performed during the W7-X experimental campaign OP1.2b, where electromagnetic features of turbulence were measured with the probe, at different magnetic configurations.

\footnotetext{
${ }^{1}$ Corresponding author.
} 


\section{Contents}

1 Introduction 1

2 The project and the diagnostic design challenges 3

2.1 Magnetic fluctuation measurements in W7-X ECRH environment 3

2.2 Realization installation and commissioning of the HRP probe head 6

3 Preliminary results examples from OP1.2b experimental campaign 6

4 Conclusions 9

\section{Introduction}

Electromagnetic filamentary turbulent structures [1-4] characterize the edge region of different magnetic configurations including Reversed Field Pinch, stellarator and tokamak [5-7]. In the latter case strong currents are associated also to Edge Localized Modes (ELM) filamentary structures [8-10]. Current associated to filaments can induce a further energy loss channel [1], furthermore high current filaments can eventually induce field line bending or modification of magnetic topology with detrimental effects on the plasma facing components $[11,12]$.

The study of those phenomena in Weldenstein-7X stellarator [13] is of particular interest as the electromagnetic features of filaments are expected to become more relevant increasing the local plasma beta [5].

To investigate the presence and features of such phenomena in the edge and Scrape-Off-Layer (SOL) region of the W7-X experiment an insertable probe head diagnostic, called High Resolution Probe (HRP), was designed and commissioned in the collaboration framework between Consorzio RFX, IPP Greifswald and Forschungszentrum Jülich. The HRP probe head aim is to provide information on different quantities: 1) investigation on the presence and the features of parallel current density associated to filamentary turbulent or ELM-like structures; 2) the time evolution of the flow radial profiles using Mach probe array is considered as a further interesting part of the study, given the strong interplay expected between the turbulent fluctuation and the average flows; 3) the radial propagation of turbulent flux can also be measured.

To provide the requested physics quantities, the probe head contains both electrostatic and magnetic sensors. In particular two groups of five electrostatic pins are placed at two different radial positions on the probe head. These groups are conceived to provide the possibility to realize the 5-pin balanced triple probe configuration [14]. The method allows measurements of electron temperature, $T_{e}$, poloidal electric field and electron density, $n_{e}$, virtually in the same location, so that also the measurement of the electrostatic turbulent particle flux can also be estimated, including the information on $T_{e}$ fluctuations. On the other hand different combination of floating potential measurements allow the estimate of $E \times B$ flow. In addition the probe head is equipped with four 
couples of pins arranged in order to provide four Mach probes placed at different radial positions, for the measurement of flow profile in direction parallel to the local magnetic filed equilibrium. The sensor layout within the probe head is shown in figure 1a. Each set of 5-pins is arranged in two rows toroidally spaced by $5.5 \mathrm{~mm}$, and within each toroidal row the pins are poloidally spaced by 6 $\mathrm{mm}$. In addition the two 5 -pin sets are radially spaced by $35 \mathrm{~mm}$. The cross-field plane including the set of tri-axial magnetic coils is $9 \mathrm{~mm}$ toroidally from the 5-pins sets. The graphite pins have a radius of $1.25 \mathrm{~mm}$ while the magnetic coils are cubic shaped, $8 \mathrm{~mm}$ side. Both electrostatic and magnetic measurements are performed with a sampling rate of $2 \mathrm{Ms} / \mathrm{s}$. All signals were collected at the same sampling frequency for the entire plunge duration so that the radial profiles are collected for the entire available sensor set. In the case of 5-pin triple probe configuration the electron density and the electron temperature within a single set are measured virtually in the same radial and poloidal position and with a small toroidal displacement of $2.7 \mathrm{~mm}$, so that in this case eventual time delays are expected to be negligible. On the other hand the relative position of magnetic sensors with respect to electrostatic pins has to be properly evaluated accordingly to the phenomena under investigation, in addition it has to be accounted for that the magnetic fluctuations have a less localized nature with respect to the electrostatic quantities.
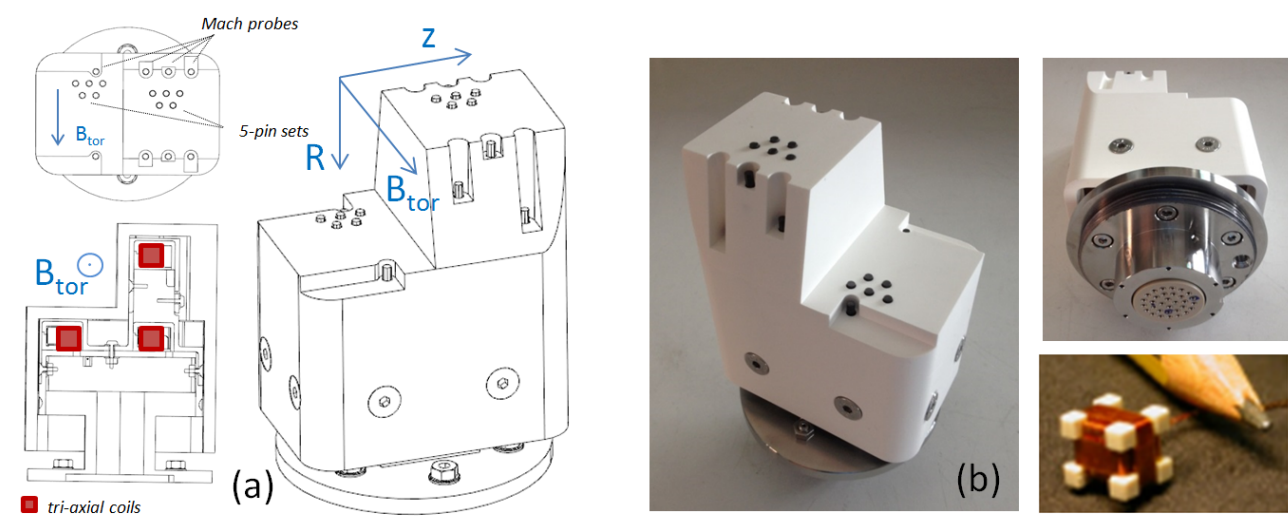

Figure 1. Schematic sensor layout within the probe head (a); HRP probe head final assembly: pictures show the probe head, the electrical feedthrough ready for the connection on the manipulator, the detail of one tri-axial coil magnetic sensor (b).

The magnetic sensors are constituted by tri-axial magnetic coils, arranged as a minimum set to define a cross-field plane. The evaluation of $J_{p a r}$ the current density parallel to the background local magnetic field, $B$, is performed according to the Ampère law $J_{\text {par }}=\frac{(\nabla \times B)_{\text {par }}}{\mu_{0}}$.

The technique is borrowed from astrophysical plasmas $[15,16]$, where it was used for the direct measurement of currents in the magnetosphere. In its simplified 2D version it was applied for the first time in a fusion experiment in RFX-mod. In this case with a probe head combining both magnetic and electrostatic pins dubbed U-probe [6]. By using the analogous diagnostic concept the estimate of the current density associated to turbulent filaments was estimated and compared in different experiments including RFX-mod in Reversed Field Pinch as well as in tokamak configuration, in Torpex device, in the stellarator TJ-II [5] and also in COMPASS tokamak [17], where a direct estimate of current density inside ELMs was provided [9]. 
The design phase of the HRP probe is described in detail in [18]. In this paper a special focus is dedicated to the diagnostic design challenges, section 2, including the theoretical and experimental evaluation of the solution adopted to perform the magnetic fluctuation measurements in the ECRH environment and the realization and commissioning of the probe head. In sec. 3 two examples of preliminary results, obtained during the $2018 \mathrm{OP} 1.2 \mathrm{~b}$ experimental campaign in W7-X, are presented.

\section{The project and the diagnostic design challenges}

The reference W7-X operation scenario considered for the probe head design was based on exploiting the available Electron Cyclotron Resonance Heating (ECRH) power up to $7 \mathrm{MW}$ for a discharge duration of 10 to $20 \mathrm{~s}$. In these conditions the stray radiation impinging on the plasma facing components is expected to be $50 \mathrm{~kW} / \mathrm{m}^{2}$ [19]. It could be considered that the probe head have to be installed on fast reciprocating manipulator and most of the time the probe head will be located in a retracted position slightly recessed behind the first wall, called the "park position". In this position, behind the first wall elements but still facing the plasma chamber, the stray radiation is expected to be reduced to $1 / 4$ factor [19].

The probe head plasma facing materials were chosen according to the specification of withstanding the heat load during operation. To this respect graphite was selected for the exposed part of electrostatic pins, while for the external case of the probe head the use of Boron Nitride was envisaged. This choice is due to minimizing spurious effects that may be caused by conducting materials on the magnetic fluctuation measurements.

It is worth mentioning that the Boron Nitride $(\mathrm{BN})$ insulating probe head case can be considered practically transparent to the $\mathrm{f}=140 \mathrm{GHz}$ ECRH stray radiation. While it helps to reduce the heat load to the probe, it also implies that the magnetic sensors needs to be protected from the same radiation. The magnetic pick-up coil sensors constitute a distinguished part of the probe head design and a specific attention is deserved to this issue. Filtering out the $\mathrm{f}=140 \mathrm{GHz}$ however has to be achieved without compromising the magnetic fluctuations measures. It was evaluated that a good compromise for the probe head turbulence measurements would be to make the magnetic sensor fluctuations available in the range of frequency up to $f_{\text {meas }}=1 \mathrm{MHz}$. The suitable shield for magnetic sensor therefore should work as a low-pass filter for the magnetic fluctuation to be measured (up to $f_{\text {meas }}=1 \mathrm{MHz}$ ), and at the same time should act as effective shield at $\mathrm{f}=140 \mathrm{GHz}$ to prevent the ECRH stray radiation damage of the sensor itself.

\subsection{Magnetic fluctuation measurements in W7-X ECRH environment}

The shield design should therefore provide enough attenuation for the $f_{E C R H}=140 \mathrm{GHz}$, allowing at the same time acceptable level of signal for the magnetic plasma fluctuations in the range of frequencies up to $f_{\text {meas }}=1 \mathrm{MHz}$. The skin depth $\delta=\sqrt{\frac{2}{\omega \mu \sigma}}$ for copper conductivity, $\sigma$, is $0.18 \mu \mathrm{m}$ at $140 \mathrm{GHz}$ and $66 \mu \mathrm{m}$ at $1 \mathrm{MHz}$. So that a copper shield of few micrometer thickness $l$, represents a thin shields $(l \ll \delta$ ) for magnetic fluctuations up to $1 \mathrm{MHz}$ while a thick one $(l \gg \delta)$ at $140 \mathrm{GHz}$. The attenuation of a closed shield at an angular frequency $\omega=2 \pi f$ is given by the modulus of the attenuation factor $S_{H}$, which according to [20] can be approximated as $S_{H}=1+\frac{\left(j \omega \sigma \mu_{0} l V\right)}{A}$ for thin shield and $S_{H}=\frac{1}{2}\left(1+\frac{V)}{\delta \mu_{r} A}+j \frac{V)}{\delta \mu_{r} A}\right) e^{(1+j) \frac{1}{\delta}}$ for a thick shield, where 
$l$ is the shield thickness, $V$ and $A$ the volume and the surface of the closed shield respectively. The estimate of the attenuation $\left|S_{H}\right|$ for the two frequencies ( $1 \mathrm{MHz}$ and $140 \mathrm{GHz}$ ), for a closed shield suitable to host the tri-axial magnetic sensor (cube-shaped, $8 \mathrm{~mm}$ side) is shown in figure 2, where the attenuation was evaluated for copper, $\sigma_{C u}=5.9 \cdot 10^{7}(\Omega \mathrm{m})^{-1}$, cubic shield, size $a=12 \mathrm{~mm}$, as a function of the shield thickness $l$.

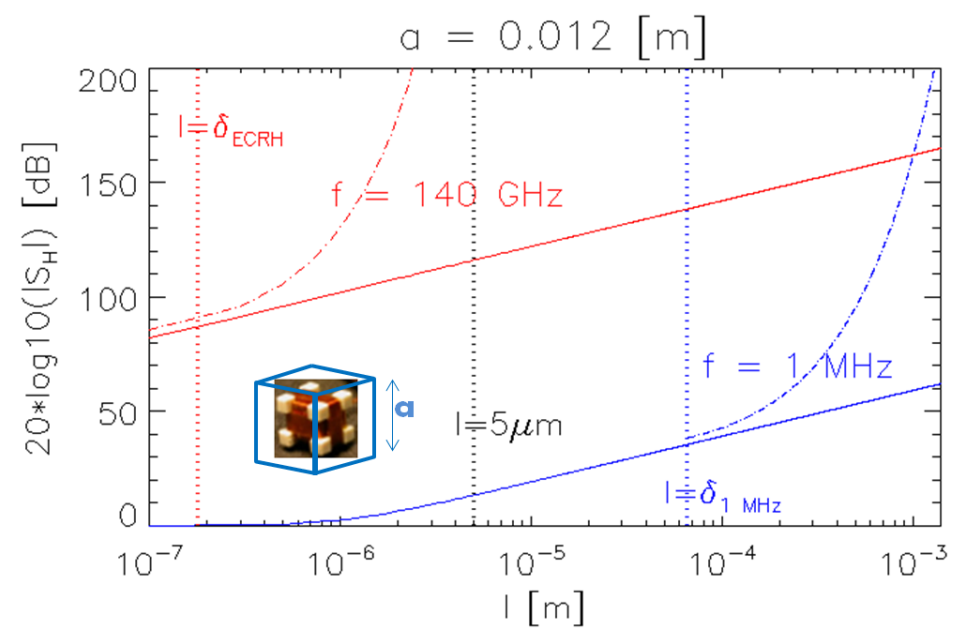

Figure 2. Analytical estimate for the attenuation expected for $f=140 \mathrm{GHz}$ (red line) and $f=1 \mathrm{MHz}$ (blue line) according to the thin (continuous line) and thick (dashed-dot line) closed shield approximations as a function of the shield thickness, the corresponding skin depths are indicated by dotted vertical lines.

The calculation was performed in order to identify the proper shield thickness value for the specific application. It can be observed that, as expected, the attenuation of the $1 \mathrm{MHz}$ radiation (blue lines) is always by far lower than the one expected for the $f_{E C R H}=140 \mathrm{GHz}$ (red lines). The results indicate that a closed copper shield thickness of the order of 3 up to $5 \mu \mathrm{m}$ (vertical dotted line), could be the suitable solution. Given this result the shield need a mechanical support for properly hosting the magnetic sensor. To this aim an insulating ceramic case was selected and the copper film was deposited on its external surface, see [18].

An experimental verification of the effectiveness of the designed shield was performed on a shield prototype. The available hardware allowed generating fluctuating magnetic field up to a frequency of $10 \mathrm{MHz}$. Larger frequencies are achievable as well, however a dedicated noise reduction experimental setup for improving the signal to noise ratio would be necessary. The experimental results are shown in figure 3. The measurements of the attenuation provided by the shield prototype are shown as a function of the source signal frequency. Experimental data, blue asterisks, are compared with analytical estimates obtained for two thickness of the copper film and for two different material conductivities. The experimental verification resulted in a slightly reduced attenuation with respect to the model, this effect can be related to either model approximations and to the eventual non-uniformities of the deposited copper film thickness on the cubic shield support edges. These results experimentally confirm that the shield plays its role successfully, with an acceptable attenuation of $-3 \mathrm{~dB}$ for measured magnetic fluctuations up to $700 \mathrm{kHz}$. In figure 4 the picture of the as built shield together with the respective locations of the three tri-axial magnetic 
coils in the probe head inner structure are shown.

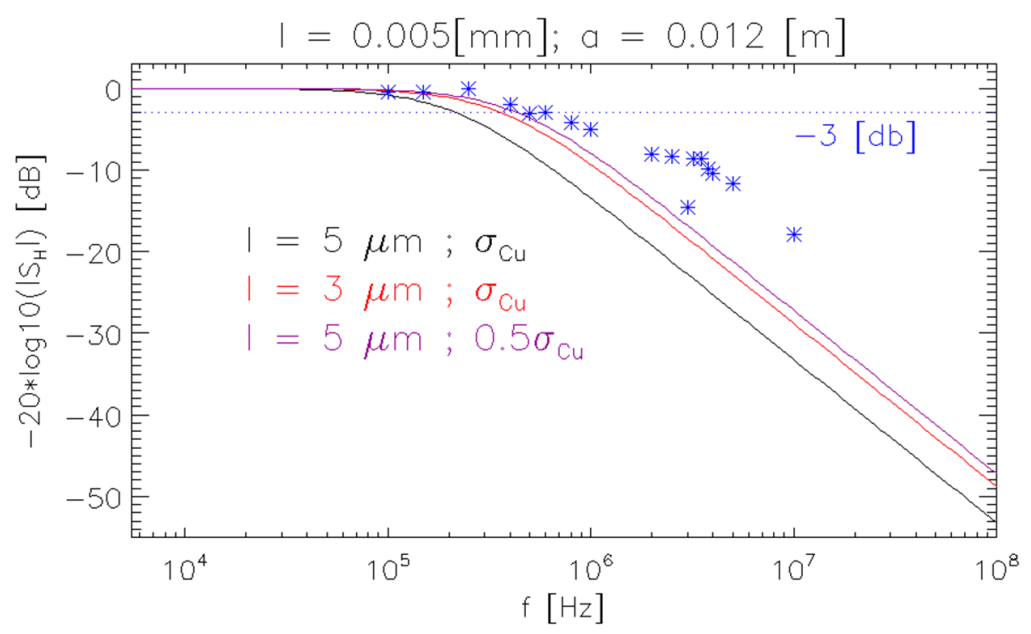

Figure 3. Experimental measurements (blue asterisks) of attenuation performed by the $l=5 \mu \mathrm{m}$ copper deposited closed cubic, $a=12 \mathrm{~mm}$, shield prototype as a function of radiation frequency. Corresponding analytical estimates (continuous lines) for different conductivities or shield thicknesses are also shown.

The internal structure of the probe head guarantee the relative positioning and electrical insulation of both graphite pins and magnetic coils inside the BN case. The chosen material was Vespel, allowed in W7-X vacuum vessel, however not for direct exposure to plasma. This design choice was supported from both thermal simulations and specifical evaluation of the behavior of the Vespel material exposed to ECRH stray radiation.

The thermal simulations were performed with the ANSYS code, to evaluate the temperature distribution on the inner structure exposed to the operation condition expected in W7-X. Two cases were simulated: application of the heat load only on the front side of the probe or with a heat load uniformly distributed. The second one resulted the worst considered scenario and is shown in figure 4. This result was obtained by applying 10 $\mathrm{kW} / \mathrm{m}^{2}$ (expected stray radiation at the probe park position) for 10 seconds (expected pulse length) on all external surfaces of the Vespel parts. In this case the peak temperature of $171{ }^{\circ} \mathrm{C}$ is reached only in some limited regions at the corners.

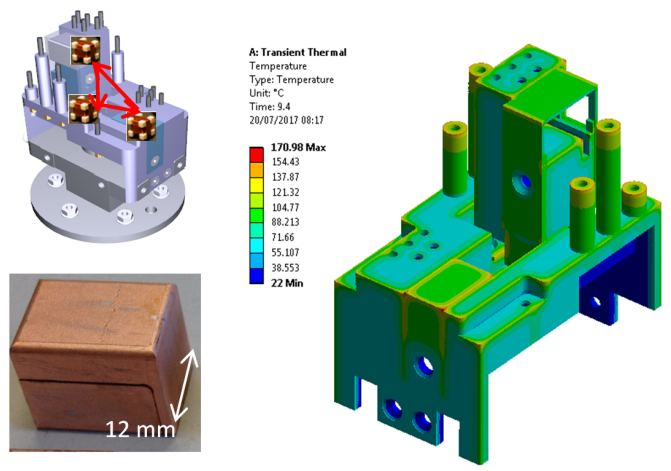

Figure 4. Left: Picture of the closed copper shield as built for each magnetic sensor and the respective location of each tri-axial coils inside the probe head. Right: temperature distribution on the inner Vespel structure as obtained from ANSYS code exposed to a homogeneous distribution of radiated power.

This temperature is considered acceptable for Vespel. In addition an evaluation of the $140 \mathrm{GHz}$ ECRH stray radiation power fraction adsorbed by Vespel was performed. The estimate was obtained accounting for the attenuation constant, 
$\alpha=\frac{1}{\delta}=100 \mathrm{~Np} / \mathrm{m} \approx 870 \mathrm{~dB}$, as measured for Vespel in [21]. In particular for the top part of the Vespel structure, the closest one to the plasma, with a thickness of $\sim 2 \mathrm{~mm}$, the total fraction of adsorbed power estimate is lower than $30 \%$, adding a further risk reduction for the probe head. As described in the following, the HRP was also used in the record discharges lasting $100 \mathrm{~s}$, approaching a fully safe maximum temperature of $100^{\circ} \mathrm{C}$ in the top part of the probe at the end of the experimental day.

\subsection{Realization installation and commissioning of the HRP probe head}

In figure 5 some phases of the assembly sequence are shown. The stainless steel interface for the connection to the manipulator, the Vespel structure and the Boron Nitride external case are visible. The detailed description of the different components of the probe head are provided in [18].

Figure $1 \mathrm{~b}$ shows the HRP probe final as-

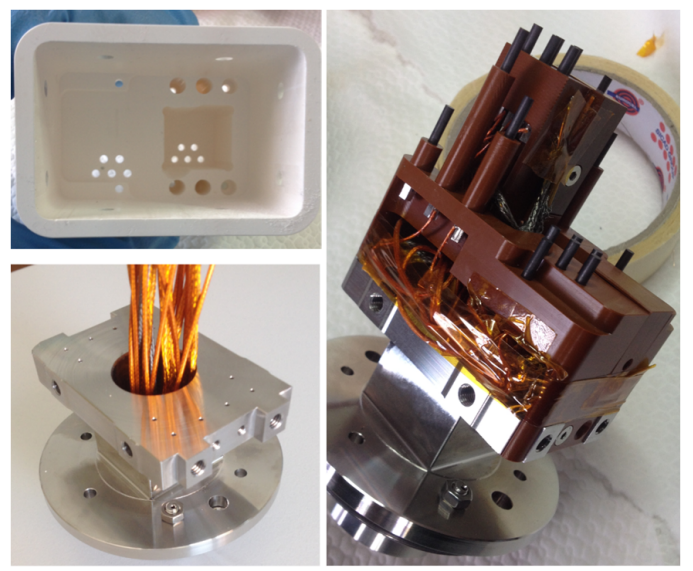

Figure 5. Representative phases of the assembly sequence of the HRP probe. sembly. The exposed to plasma part of the graphite pins are visible, arranged in two 5-pin triple probe sets and a set composed by four radially distributed Mach probes. The magnetic sensors, one example is shown in the insert, are protected by the Boron Nitride case and not visible in the picture. The detail of the electrical feedthrough for the connection to the Multi Purpose Manipulator (MPM) is also shown in the picture. The MPM fast reciprocating manipulator [22] is conceived to obtain an easy change of the probe head and allows the fast insertion and retraction, "plunge", of different diagnostics probe heads [23]. The maximum acceleration applied was $30 \mathrm{~m} / \mathrm{s}^{2}$, with the maximum insertion speed of $3.5 \mathrm{~m} / \mathrm{s}$. These constraint guided the special attention devoted to the mechanical robustness of the connection of the BN ceramic case to the stainless steel interface. Figure 6a shows the installation phase of the HRP on the manipulator. As an example, the poloidal component of magnetic fluctuations collected during a long lasting plunge is shown in Figure 6b. The largest signals observed in the magnetic fluctuations around $2.95 \mathrm{~s}$ and $3.65 \mathrm{~s}$ correspond to the deepest position programmed for the plunge in this shot $\left(R_{\text {plunge }}=6055 \mathrm{~mm}\right)$, while the longer stay is programmed on purpose in this case in a slightly recessed position $\left(R_{\text {plunge }}=6070 \mathrm{~mm}\right)$.

\section{Preliminary results examples from OP1.2b experimental campaign}

The HRP probe head was commissioned and used for the first time at the beginning of the OP1.2b, W7-X experimental campaign carried on in 2018, during iota tuning experiments [24]. The experiment was aimed to identify the suitable calibration for the W7-X current in planar coils, $I_{P C}$, in order to obtain the as designed island divertor configuration [13]. To this aim a set of subsequent identical $3.5 \mathrm{~s}, P_{E C R H}=2 M W$, discharges with different values of $I_{P C}$, ranging from 0 to $-2000 \mathrm{~A}$, were performed. The details of the experiment is described in [24], for what concerns 

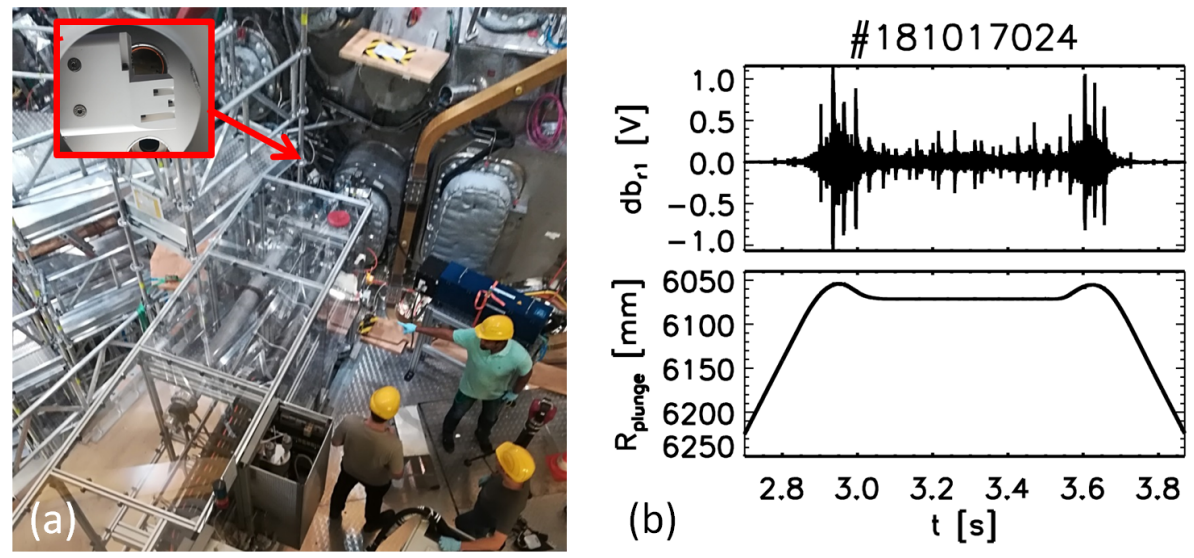

Figure 6. W7-X experimental hall: installation of the HRP probe on the MPM manipulator (a). Example of magnetic signal fluctuation, poloidal component, collected during a plunge lasting about $1 \mathrm{~s}$ in the plasma (b).
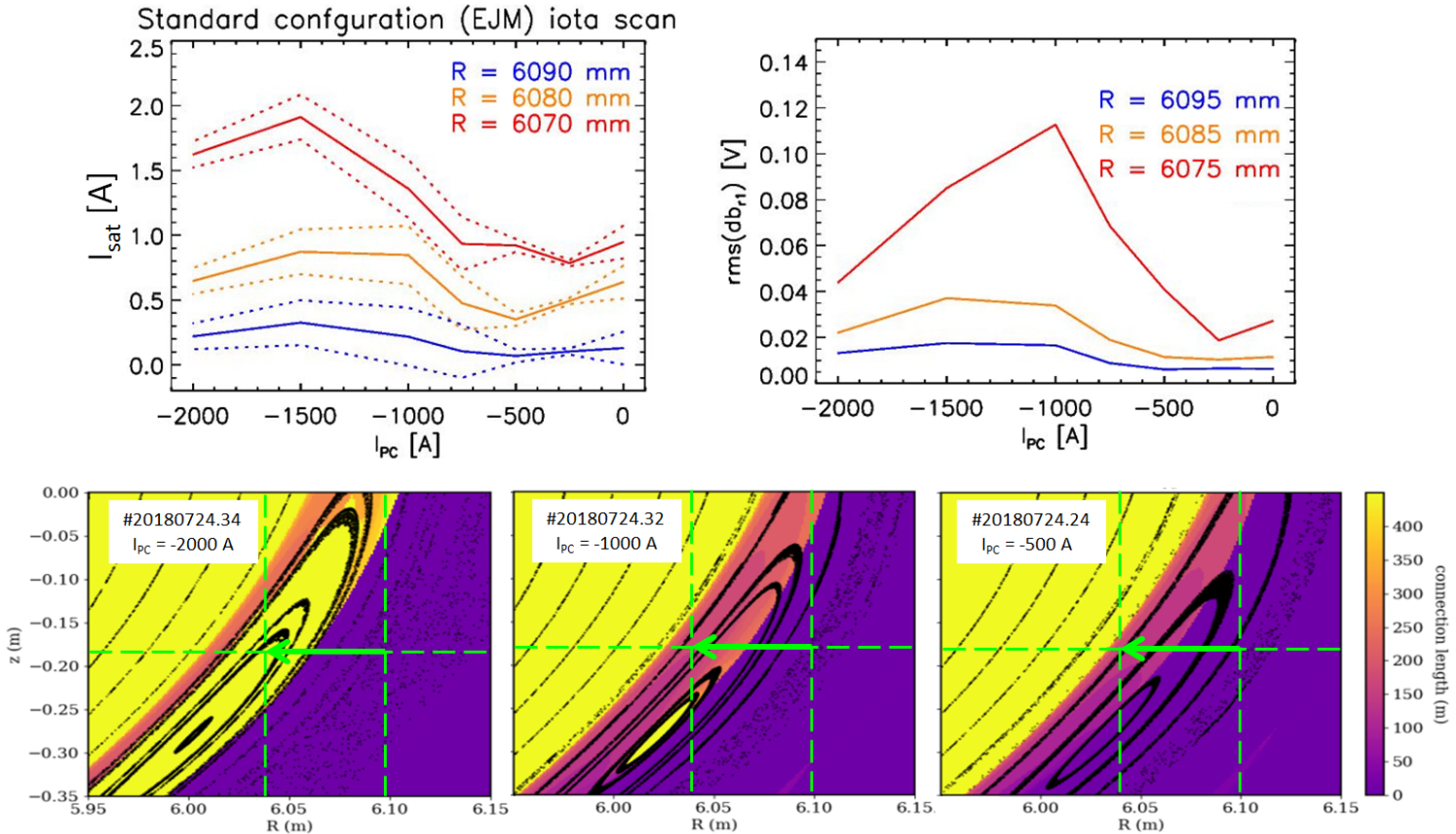

Figure 7. Top panels: profiles of $I_{\text {sat }}$ as a function of $I_{P C}$ for three different radial positions, dashed lines represent the respective standard deviation; analogous measurements for the $\delta b_{r}$. Bottom panels: Poincarè reconstruction of the magnetic topology at the MPM toroidal section, color scale indicates the local magnetic connection length map, green arrows indicates the range explored by the probe plunge. Data are shown for three different $I_{P C}$ values. 
the HRP probe measurements it is enough to say that the local magnetic topology facing the MPM location changes as a function of the applied $I_{P C}$ current. Three representative examples are shown in figure 7: the three panels in the bottom show the magnetic flux surfaces corresponding to the toroidal section of the MPM, color scales indicates the corresponding magnetic connection length map. In particular the yellow regions indicate the confined plasma, while the purple one the Scrape-Off-Layer. In the intermediate region a magnetic island is visible in the field line tracing map, however while decreasing the $I_{P C}$, from right to left in the figure, an edge confined plasma island appears, not directly connected to the main plasma column. The green arrows indicate the range respectively explored by the HRP during the MPM plunge. Each shot in the series was performed for a given $I_{P C}$ value. For each shot the radial profile of different quantities is collected during the plunge, estimated as average values performed on $1 \mathrm{~ms}$ time window. In the top-left panel the measured profiles of ion saturation current, $I_{s a t}$, that can be considered a proxy of local plasma density, at three different radial positions are shown as a function of $I_{P C}$. A tendency to increased density values is observed in correspondence to the appearance of the island. This results is consistent with the picture of the probe head entering into a confined plasma region. Analogously the profiles of the root-mean-square radial component of magnetic field fluctuation, $d b_{r 1}$, taken as representative component, are shown in the top-right panel. Also in this case the local magnetic topology modifications induced by the $I_{P C}$ consistently affect the local magnetic fluctuations.

A second result example obtained with the HRP probe is shown in figure 8 . The time window refers in this case to the phase, see figure $6 \mathrm{~b}$, of the plunge staying in the deep position, $R=6.07 \mathrm{~m}$, corresponding to the near Scrape-Off-Layer in this case. This procedure allows a more detailed monitoring of fluctuations. Data were collected in the so called "limiter" magnetic configuration. This configuration differently from the standard one does not exhibits the five edge magnetic islands outside of the confined plasma column. In these conditions a clearly correlated bursty behavior, also seen in other diagnostics [25], is observed both in electrostatic and magnetic fluctuations. In particular figure 8 shows that this bursty behavior is associated to abrupt increases in the power spectrum involving the whole range of explored frequencies, as shown in the example

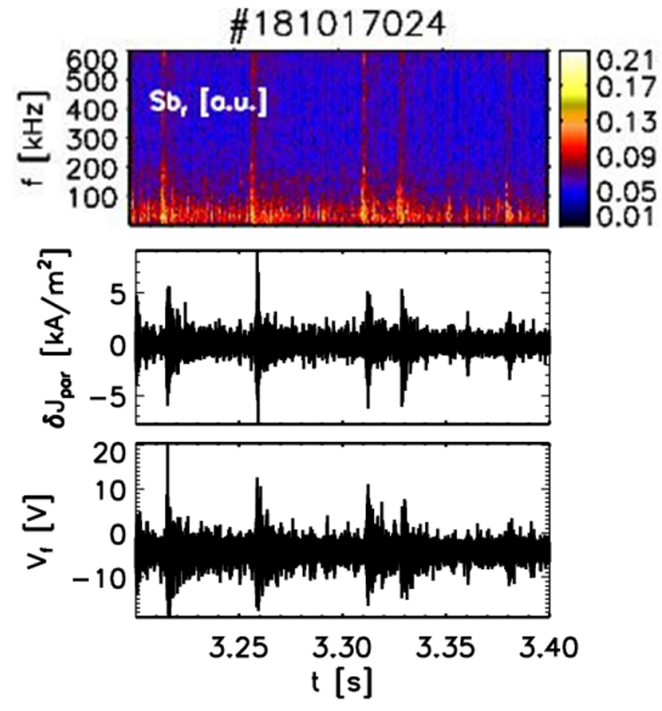

Figure 8. From top to bottom: time evolution during the bursty phase of $\delta b_{r}$ spectrogram, parallel current density fluctuations, $\delta J_{\text {par }}$, floating potential, $V_{f}$, as measured by the HRP probe. of $d b_{r}$ spectrograms in the top panel. This behavior is indicative of presence of localized structures, or filaments, better than global modes. In addition exploiting the 2D array of tri-axial magnetic coils the direct measurement of current density was performed, middle panel in figure 8 , revealing that the bursts are clearly associated to parallel current density peaks, so that they can be considered as current carrying filaments. This results is reminiscent [9] of similar filamentary behavior observed in tokamak ELMs. 


\section{Conclusions}

The HRP was successfully operated during W7-X OP1.2b experimental campaign, in discharges with maximum power ECRH (6 MW) with the probe head plunged inside the edge islands and a maximum program duration of $100 \mathrm{~s}$, with the probe in the park position. Typical plunge duration was $0.6 \mathrm{~s}$ and up to four plunges per program were applied. In some cases the probe was positioned to remain in the SOL for up to $2.5 \mathrm{~s}$, which did not pose any heat load problem. It is worth noting that the sensor sets of the HRP probe head could not be fully exploited at this stage, due to a technical issue occurred on the MPM interface. Nevertheless both electrostatic and magnetic fluctuation signals were measured, including the direct measurement of parallel current density fluctuations. The first results provided consistent modifications of edge parameters during iotatuning experiments and example evidence of the presence of current carrying filaments in the edge region.

\section{Acknowledgments}

This work has been carried out within the framework of the EUROfusion Consortium and has received funding from the Euratom research and training programme 2014-2018 and 2019-2020 under grant agreement No 633053. The views and opinions expressed herein do not necessarily reflect those of the European Commission.

\section{References}

[1] DIppolito D, Myra J and Zweben S 2011 Physics of Plasmas 18060501

[2] Krasheninnikov S I 2001 Physics Letters A 283 368-370

[3] Myra J 2007 Physics of Plasmas 14102314

[4] Migliucci P, Naulin V and Contributors J E 2010 Physics of Plasmas 17072507

[5] Spolaore M, Vianello N, Furno I, Carralero D, Agostini M, Alonso J, Avino F, Cavazzana R, De Masi G, Fasoli A et al. 2015 Physics of Plasmas 22012310

[6] Spolaore M, Vianello N, Agostini M, Cavazzana R, Martines E, Scarin P, Serianni G, Spada E, Zuin M and Antoni V 2009 Physical review letters 102165001

[7] Furno I, Spolaore M, Theiler C, Vianello N, Cavazzana R and Fasoli A 2011 Physical review letters 106245001

[8] Kirk A, Koch B, Scannell R, Wilson H, Counsell G, Dowling J, Herrmann A, Martin R, Walsh M et al. 2006 Physical review letters 96185001

[9] Spolaore M, Kovařík K, Stöckel J, Adamek J, Dejarnac R, Ďuran I, Komm M, Markovic T, Martines E, Panek R et al. 2017 Nuclear Materials and Energy 12 844-851

[10] Vianello N, Naulin V, Schrittwieser R, Müller H, Zuin M, Ionita C, Rasmussen J, Mehlmann F, Rohde V and Team A U 2011 Physical review letters 106125002

[11] Hoare D, Militello F, Omotani J, Riva F, Newton S, Nicholas T, Ryan D and Walkden N 2019 PPCF submitted

[12] Wingen A, Evans T, Lasnier C and Spatschek K 2010 Physical review letters 104175001 
[13] Klinger T, Andreeva T, Bozhenkov S A, Brandt C, Burhenn R, Buttenschön B, Fuchert G, Geiger B, Grulke O, Laqua H P et al. 2019 Nuclear Fusion

[14] Tsui H, Bengtson R, Li G, Lin H, Meier M, Ritz C P and Wootton A 1992 Review of scientific instruments 63 4608-4610

[15] Van Allen J A 2006 American journal of physics 74 809-814

[16] Martines E, Vianello N, Sundkvist D, Spolaore M, Zuin M, Agostini M, Antoni V, Cavazzana R, Ionita C, Maraschek M et al. 2009 Plasma Physics and Controlled Fusion 51124053

[17] Kovarik K, Duran I, Stockel J, Seidl J, Adamek J, Spolaore M, Vianello N, Hacek P, Hron M and Panek R 2017 Review of Scientific Instruments $\mathbf{8 8} 035106$

[18] Agostinetti P, Spolaore M, Brombin M, Cervaro V, Franchin L, Grulke O, Killer C, Martines E, Moresco M, Peruzzo S et al. 2018 IEEE Transactions on Plasma Science 46 1306-1311

[19] Hathiramani D, Binder R, Brakel R, Broszat T, Brucker B, Cardella A, Endler M, Grosser K, Hirsch M, Laqua H et al. 2013 Fusion Engineering and Design 88 1232-1235

[20] Tesche F M, Ianoz M and Karlsson T 1996 EMC analysis methods and computational models (John Wiley \& Sons)

[21] Pastol Y, Arjavalingam G, Halbout J M and Kopcsay G 1989 Electronics Letters 25 523-524

[22] Nicolai D, Borsuk V, Drews P, Grulke O, Hollfeld K, Krings T, Liang Y, Linsmeier C, Neubauer O, Satheeswaran G et al. 2017 Fusion Engineering and Design 123 960-964

[23] Killer C, Grulke O, Drews P, Gao Y, Jakubowski M, Knieps A, Nicolai D, Niemann H, Sitjes A P and Satheeswaran G 2019 Nuclear Fusion

[24] Samuel A Lazerson1 e a 2019 Nuclear Fusion, submitted

[25] Wurden G A et al. 2019 46th EPS Conference Milan, Italy July 8-12, P2.1068 